\title{
Development of a newborn screening follow-up algorithm for the diagnosis of isobutyryl-CoA dehydrogenase deficiency
}

Devin Oglesbee, $\mathrm{PhD}^{1}$, Miao He, $\mathrm{PhD}^{4}$, Nilanjana Majumder ${ }^{4}$, Jerry Vockley, MD, PhD ${ }^{4}$, Ayesha Ahmad, $M D^{5}$, Brad Angle, $M D^{6}$, Barbara Burton, $M D^{6}$, Joel Charrow, $M D^{6}$, Regina Ensenauer, $M D^{1,7}$, Can H. Ficicioglu, $M D, P h D^{8}$, Laura Davis Keppen, $M D^{9}$, Deborah Marsden, $M D^{10}$, Silvia Tortorelli, $M D, P h D^{1,2}$, Si Houn Hahn, MD, PhD ${ }^{1,2,3}$, and Dietrich Matern, $M D^{1,2,3}$

\begin{abstract}
Purpose: Isobutyryl-CoA dehydrogenase deficiency is a defect in valine metabolism and was first reported in a child with cardiomyopathy, anemia, and secondary carnitine deficiency. We identified 13 isobutyryl-CoA dehydrogenasedeficient patients through newborn screening due to an elevation of $\mathrm{C}_{4}$-acylcarnitine in dried blood spots. Because $\mathrm{C}_{4}$-acylcarnitine represents both isobutyryl- and butyrylcarnitine, elevations are not specific for isobutyryl-CoA dehydrogenase deficiency but are also observed in short-chain acyl-CoA dehydrogenase deficiency. To delineate the correct diagnosis, we have developed a follow-up algorithm for abnormal $\mathrm{C}_{4}$-acylcarnitine newborn screening results based on the comparison of biomarkers for both conditions. Methods: Fibroblast cultures were established from infants with $\mathrm{C}_{4}$-acylcarnitine elevations, and the analysis of in vitro acylcarnitine profiles provided confirmation of either isobutyryl-CoA dehydrogenase or short-chain acyl-CoA dehydrogenase deficiency. Isobutyryl-CoA dehydrogenase deficiency was further confirmed by molecular genetic analysis of the gene encoding isobutyryl-CoA dehydrogenase (ACAD8). Plasma acylcarnitines, urine acylglycines, organic acids, and urine acylcarnitine results were compared between isobutyryl-CoA dehydrogenase- and short-chain acyl-CoA dehydrogenase-deficient patients. Results: Quantification of $\mathrm{C}_{4}$-acylcarnitine in plasma and urine as well as ethylmalonic acid in urine allows the differentiation of isobutyryl-CoA dehydrogenase-deficient from short-chain acyl-CoA dehydrogenase-deficient cases. In nine unrelated patients with isobutyryl-CoA dehydrogenase deficiency, 10 missense mutations were identified in ACAD8. To date, 10 of the 13 isobutyryl-CoA dehydrogenase-deficient patients remain asymptomatic, two were lost to follow-up, and one patient required frequent hospitalizations due to emesis and dehydration but is developing normally at 5 years of age. Conclusion: Although the natural history of isobutyryl-CoA dehydrogenase deficiency must be further defined, we have developed an algorithm for rapid laboratory evaluation of neonates with an isolated elevation of $\mathrm{C}_{4}$-acylcarnitine identified through newborn screening. Genet Med 2007:9(2):108-116.
\end{abstract}

Key Words: ACAD8, acylcarnitine analysis, newborn screening, isobutyryl-CoA dehydrogenase deficiency, tandem mass spectrometry

Isobutyryl-CoA dehydrogenase (IBD) is a recently characterized enzyme that catalyzes the third step in the degradation of the branched chain amino acid valine (Fig. 1). ${ }^{1}$ It is encoded by ACAD8 located on chromosome 11q25 (OMIM *604773). Nine patients with IBD deficiency have been reported to date. ${ }^{2-5}$ The first patient presented with dilated cardiomyopathy, anemia, and carnitine deficiency. ${ }^{2}$ An elevated $\mathrm{C}_{4}$-acylcarnitine was noted in a plasma acylcarnitine profile, but a subse-

From the Departments of ${ }^{1}$ Laboratory Medicine and Pathology, ${ }^{2}$ Medical Genetics, and ${ }^{3}$ Pediatric and Adolescent Medicine, Mayo Clinic College of Medicine, Rochester, Minnesota; ${ }^{4}$ Children's Hospital of Pittsburgh, Pittsburgh, Pennsylvania; ${ }^{5}$ Children's Hospital of Michigan, Detroit, Michigan; ${ }^{6}$ Children's Memorial Hospital, Chicago, Illinois; ${ }^{7}$ Dr. von Hauner Children's Hospital, Ludwig-Maximilians University, Munich, Germany; ${ }^{8}$ Children's Hospital of Philadelphia, Philadelphia, Pennsylvania; ${ }^{9}$ Sanford Children's Specialty Clinic, Sioux Falls, South Dakota; ${ }^{10}$ Children's Hospital, Boston, Massachusetts. quent urine organic acid analysis was normal. Treatment with oral L-carnitine supplementation led to catch-up growth and normalization of the cardiac status. She remains carnitine dependent at almost 11 years of age (Dr. Stephen Cederbaum, 2006, Mental Retardation Research Center, UCLA, Los Angeles, CA, personal communication). The other eight patients were identified by newborn screening when isolated elevations of $\mathrm{C}_{4}$-acylcarnitine prompted further diagnostic investiga-

\footnotetext{
Dietrich Matern, MD, Biochemical Genetics Laboratory, Mayo Clinic, 200 First Street SW, Rochester, MN 55905; E-mail: dietrich.matern@mayo.edu

The authors declare no conflict of interest.

Submitted for publication September 22, 2006

Accepted for publication November 27, 2006.

DOI: 10.1097/GIM.0b013e31802f78d6
} 

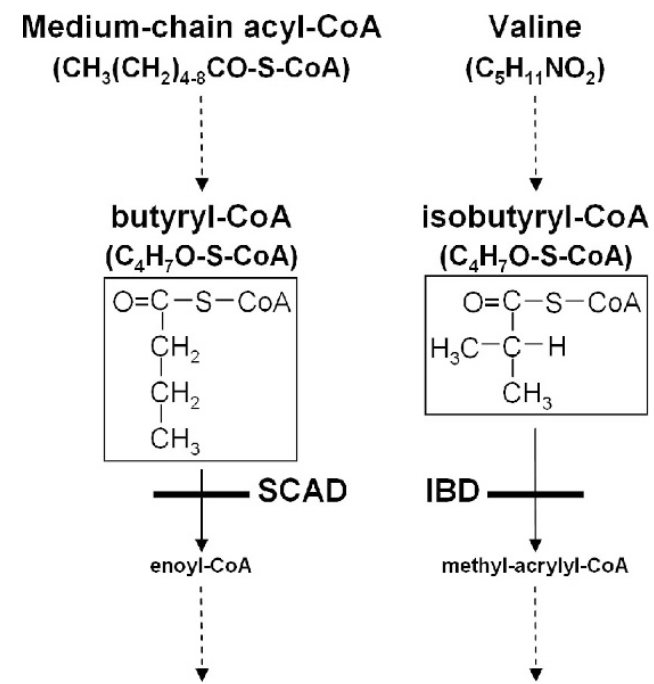

Fig. 1. Metabolic pathways resulting in butyryl- and isobutyryl-CoA intermediates. Selected chemical structures and formulas are provided to demonstrate the mass equivalence between these isomers. SCAD, short-chain acyl-CoA dehydrogenase; IBD, isobutyryl-CoA dehydrogenase.

tions. For six patients, clinical information is available. Two patients have remained asymptomatic for more than 1 year without treatment ${ }^{4,5}$; one patient was noted to have muscle hypotonia and mild developmental delay at 8 months of age ${ }^{4}$; two patients were treated for speech delays at 5 years and 2 years of age, respectively ${ }^{5}$, but have normal growth and development; and one patient was incidentally noted at 1 year of age to have mild branch peripheral pulmonary stenosis, which was detected by echocardiography to rule out a cardiomyopathy. ${ }^{5}$

Although these case reports are not conclusive regarding the clinical relevance of IBD deficiency, they support the notion that it is detectable by newborn screening through blood spot acylcarnitine analysis by tandem mass spectrometry. ${ }^{6}$ However, $\mathrm{C}_{4}$-acylcarnitine is not a specific marker for IBD deficiency as it does not represent isobutyrylcarnitine alone, but it is a mixture of isobutyryl- and butyrylcarnitine. The latter is an intermediate of short-chain fatty acid $\beta$-oxidation and a marker for short-chain acyl-CoA dehydrogenase (SCAD; E.C. 1.3.99.2) deficiency (OMIM \#201470) (Fig. 1).

Similar to IBD deficiency, the clinical phenotype of SCAD deficiency remains poorly defined, although it was first described almost two decades ago. ${ }^{7}$ The first two patients reported with SCAD deficiency presented during the first week of life with poor feeding, vomiting, metabolic acidosis, and lethargy. One patient died within 6 days, and the other recovered completely without any further such episodes. Symptoms described in other SCADdeficient patients include muscle weakness, developmental delay, seizures, respiratory distress, congenital anomalies, dysmorphism, and maternal pregnancy complications. However, asymptomatic cases have also been described.8,9 The biochemical phenotype of SCAD deficiency has been delineated through clinical testing and includes persistent ethylmalonic aciduria with or without the overexcretion of methylsuccinic acid and accumulation of butyrylcarnitine in plasma and blood. The molecular genetics of SCAD deficiency is complicated by two SCAD gene $(A C A D S)$ variants $(511 \mathrm{C}>\mathrm{T}, 625 \mathrm{G}>\mathrm{A})$ of questionable clinical importance. These variants do not abolish SCAD activity and occur in homozygous or compound heterozygous states in up to 7\% of the general population..$^{10-14}$ When ACADS mutations that have been shown to lead to complete loss of SCAD activity in vitro are identified in patients with biochemical evidence of SCAD deficiency, the diagnosis is confirmed. However, some individuals with both biochemical and molecular genetic evidence of SCAD deficiency have been reported to remain asymptomatic. ${ }^{8,9}$

The variable expression of disease in both IBD and SCAD deficiency have led to the designation of these conditions as secondary targets in the newborn screening panel recently recommended by a working group of the American College of Medical Genetics contracted by the Human Resources and Services Administration of the U.S. Department of Health and Human Services. ${ }^{15}$ This work group was charged to develop recommendations for which disorders should be included in every newborn screening program to overcome inequalities between the various state-mandated newborn screening panels. The concept of secondary targets is based on the inevitable identification of conditions (such as IBD and SCAD deficiency) whose characteristic metabolites are readily identified in conjunction with other conditions designated as primary targets.

To develop an efficient and least invasive follow-up algorithm for abnormal $\mathrm{C}_{4}$-acylcarnitine newborn screening results, we compared biomarkers between cohorts of IBD-deficient and SCAD-deficient patients after confirmation of the correct diagnosis by biochemical and/or molecular genetic analyses.

\section{MATERIALS AND METHODS}

\section{Patients and controls}

Thirteen cases (11 families) of IBD deficiency were identified through newborn screening in Illinois, Massachusetts, Michigan, Minnesota, Pennsylvania, and South Dakota between 2001 and 2006. The IBD-deficient patients came to our attention either through newborn screening provided by our laboratory or by referral of patient samples for follow-up of abnormal newborn screening results obtained elsewhere. A cohort of 18 SCAD-deficient cases also identified by newborn screening was used to compare laboratory findings. The Mayo Clinic and University of Pittsburgh Institutional Review Boards approved this project.

\section{Clinical evaluation}

Clinical details regarding the IBD-deficient cases' current health status were collected whenever possible from genetic centers in the states from which diagnostic referrals were sent. A summary of the patients' clinical conditions is listed in Table 1. 
Table 1

Genotypes, clinical notes, and ancestry of IBD-deficient cases

\begin{tabular}{|c|c|c|c|c|c|c|c|}
\hline Patient & Allele 1 & Allele 2 & Potential splice-site alterations & SCAD gene variants & Clinical condition & Gender & Ancestry \\
\hline 1 & $988 \mathrm{C}>\mathrm{T}(\mathrm{R} 308 \mathrm{~W})$ & $988 \mathrm{C}>\mathrm{T}(\mathrm{R} 308 \mathrm{~W})$ & None & None & Emesis/dehydration & Female & North African \\
\hline 2 & $289 \mathrm{G}>\mathrm{A}(\mathrm{G} 75 \mathrm{R})$ & $455 \mathrm{~T}>\mathrm{C}(\mathrm{M} 130 \mathrm{~T})$ & Exon $3(+3 \mathrm{~A}>\mathrm{G})$ & $625 \mathrm{G}>\mathrm{A} / 625 \mathrm{G}>\mathrm{A}$ & Unremarkable & Female & European \\
\hline $3 a^{a}$ & $867 \mathrm{C}>\mathrm{A}(\mathrm{H} 267 \mathrm{Q})$ & $867 \mathrm{C}>\mathrm{A}(\mathrm{H} 267 \mathrm{Q})$ & None & None & Unremarkable & Male & East Indian \\
\hline $3 b^{a}$ & $867 \mathrm{C}>\mathrm{A}(\mathrm{H} 267 \mathrm{Q})$ & $867 \mathrm{C}>\mathrm{A}(\mathrm{H} 267 \mathrm{Q})$ & None & ND & Unremarkable & Female & East Indian \\
\hline 4 & $443 \mathrm{C}>\mathrm{T}(\mathrm{P} 126 \mathrm{~L})$ & $455 \mathrm{~T}>\mathrm{C}(\mathrm{M} 130 \mathrm{~T})$ & Exon $3(+3 A>G)$ & None & Unremarkable & Female & European \\
\hline 5 & ND & ND & ND & ND & ND & Female & European \\
\hline 6 & $958 \mathrm{G}>\mathrm{A}(\mathrm{A} 298 \mathrm{~T})$ & $1129 \mathrm{G}>\mathrm{A}(\mathrm{G} 355 \mathrm{~S})$ & None & ND & ND & Female & European \\
\hline $7 \mathrm{a}^{a}$ & $687 \mathrm{~T}>\mathrm{G}(\mathrm{F} 207 \mathrm{~L})$ & $1129 \mathrm{G}>\mathrm{A}(\mathrm{G} 355 \mathrm{~S})$ & None & $\mathrm{ND}$ & Unremarkable & Male & Hispanic \\
\hline $7 b^{a}$ & ND & ND & ND & $\mathrm{ND}$ & Unremarkable & Male & Hispanic \\
\hline 8 & $512 \mathrm{C}>\mathrm{G}(\mathrm{S} 149 \mathrm{C})$ & $455 \mathrm{~T}>\mathrm{C}(\mathrm{M} 130 \mathrm{~T})$ & Exon $3(+3 A>G)$ & $625 \mathrm{G}>\mathrm{A} / 625 \mathrm{G}>\mathrm{A}$ & Unremarkable & Male & European \\
\hline 9 & $233 \mathrm{~T}>\mathrm{C}(\mathrm{M} 56 \mathrm{~T})$ & $233 \mathrm{~T}>\mathrm{C}(\mathrm{M} 56 \mathrm{~T})$ & None & $\mathrm{ND}$ & Unremarkable & Male & Hispanic \\
\hline 10 & ND & ND & ND & ND & Unremarkable & Female & Middle Eastern \\
\hline 11 & $1129 \mathrm{G}>\mathrm{A}(\mathrm{G} 355 \mathrm{~S})$ & None & None & $\mathrm{ND}$ & $b$ & Male & African American \\
\hline 12 & ND & ND & ND & $\mathrm{ND}$ & Unremarkable & Male & European \\
\hline
\end{tabular}

${ }^{a}$ Siblings.

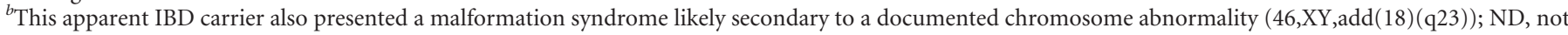
determined for lack of specimen.

IBD, isobutyryl-CoA dehydrogenase; SCAD, short-chain acyl-CoA dehydrogenase.

\section{Plasma acylcarnitine, urine organic acid, urine acylglycine, and urine acylcarnitine analyses}

Acylcarnitine analyses in dried blood spot, plasma, and urine as well as urine organic acid and acylglycine analyses were performed following established procedures. ${ }^{16,17}$

\section{Fibroblast culture and in vitro probe analysis}

Fibroblast cultures from the patients, a healthy control, an IBD-deficient control, and a SCAD-deficient control were grown in triplicate in six-well cell culture plates to approximately $90 \%$ confluence. Culture medium was replaced with $1.5 \mathrm{~mL}$ of modified minimal essential medium (Modified RPMI 1640 with L-glutamine and without isoleucine and valine; JRH Biosciences, Lenexa, KS). This incubation medium contains fatty acid-free bovine serum albumin (200 mg/dL BSA), palmitic acid $(0.2 \mathrm{mmol} / \mathrm{L})$, L-carnitine $(0.4 \mathrm{mmol} / \mathrm{L}$; Sigma, St. Louis, MO), as well as $0.8 \mathrm{mmol} / \mathrm{L} \mathrm{U}_{-}{ }^{13} \mathrm{C}$-L-valine and $\mathrm{U}^{13}{ }^{13} \mathrm{C}-\mathrm{L}-$ isoleucine (Cambridge Isotope Laboratories, Cambridge, MA). After 72 hours of incubation at $37^{\circ} \mathrm{C}$, the medium was collected for acylcarnitine analysis. Remaining cells were gently washed in phosphate-buffered saline (Dulbecco's PBS; Gibco Invitrogen, Carlsbad, CA), harvested by trypsinization $(0.25 \mathrm{~mL} 0.25 \%$ trypsin-ethylenediaminetetraacetic acid; Sigma-Aldrich, St. Louis, $\mathrm{MO}$ ), and protein content was measured following sonication using a method described by Lowry et al. ${ }^{18}$ Concentrations of butyryl- and isobutyrylcarnitine in the cell medium were normalized to cell protein concentrations.

\section{Molecular genetic analyses}

Molecular genetic analysis of $A C A D 8$ was performed using genomic DNA extracted from either fibroblasts samples or from blood spots. All 10 exons of ACAD8 and 18 intron-exon junctions were amplified by polymerase chain reaction (PCR), and DNA analysis was performed by dye-terminator sequencing PCR-amplified fragments on an Applied Biosystems 3730 DNA Analyzer (Foster City, CA) at the University of Pittsburgh Genomics and Proteomics Core Laboratory. Primer sequences for exon amplification and sequencing are available on request. ACADS molecular genetic analysis was performed by PCR amplification of 10 exons and all intronic splice-site regions with genomic DNA extracted from patient fibroblast samples or from blood spots. DNA sequences of PCR-amplified fragments were obtained through dye-terminator sequencing on an Applied Biosystems 3730 DNA Analyzer at the Mayo Clinic Molecular Genetics Laboratory. Sequence analysis was done with Mutation Surveyor software (SoftGenetics, State College, PA). Primer sequences for exon amplification and sequencing are available on request.

\section{Statistical analysis}

Recursive partitioning was used to find the optimal values for urine $\mathrm{C}_{4}$-acylcarnitine and the ratio of urine $\mathrm{C}_{4}$ - to $\mathrm{C}_{3}$ acylcarnitine to discriminate among normal, SCAD-deficient, and IBD-deficient patients. ${ }^{19}$

\section{RESULTS}

\section{Patients and controls}

Thirty-two infants came to our attention through newborn screening provided by our laboratory or through submission of samples for follow-up of abnormal newborn screening results obtained elsewhere. Thirteen cases (11 families) were 
confirmed as described below to be affected with IBD deficiency, 18 cases with SCAD deficiency, and one to be an IBD carrier. Seven IBD-deficient patients were female and six patients were male (Table 1). The geographic distribution of IBD deficiency seems widespread as six infants were European, three Hispanic, two East Indian, and the remaining two cases were of North African and Middle Eastern ancestry, respectively (Table 1).

\section{Acylcarnitines in newborn screening blood spots and plasma samples}

Twelve of 13 IBD-deficient patients were identified directly by newborn screening and referred for follow-up because of isolated elevations of $\mathrm{C}_{4}$-acylcarnitine, which ranged from 1.8 to $2.9 \mu \mathrm{mol} / \mathrm{L}$ (median, $2.1 \mu \mathrm{mol} / \mathrm{L}$; reference range: $<1.5$ $\mu \mathrm{mol} / \mathrm{L}$; Table 2). One 3 -year-old healthy sibling was tested after the newborn screening result of their younger brother (Patient 7b, Table 2). Plasma acylcarnitine analysis performed as part of follow-up investigations revealed isolated elevations of $\mathrm{C}_{4}$-acylcarnitine (range: $1.14-10.00 \mu \mathrm{mol} / \mathrm{L}$; median: 1.52 $\mu \mathrm{mol} / \mathrm{L}$; reference range: $<1.06 \mu \mathrm{mol} / \mathrm{L}$; Table 2) in all IBDdeficient cases. Similar values for $\mathrm{C}_{4}$-acylcarnitine in newborn screening blood spot and plasma samples were observed in the 18 SCAD-deficient cases (Fig. 2, A and B; Table 3).

\section{Urine acylglycines and urine organic acids}

Isobutyrylglycine levels were normal in all but one (Patient 10 in Table 2) of the 11 available urine samples from IBDdeficient patients, albeit the excretion was higher than in SCAD-deficient cases. The excretion of EMA was normal in all infants with IBD deficiency, whereas it was elevated in all SCAD-deficient cases (Tables 2 and 3, Fig. 2, C and D).

\section{In vitro probe analysis of cultured fibroblasts}

To determine the correct diagnosis, fibroblast cultures were established from small skin biopsies for confirmatory testing by in vitro studies of both the valine degradation and fatty acid oxidation pathways. All available cell lines $(n=9)$ of patients with elevated $\mathrm{C}_{4}$-acylcarnitine concentrations in newborn screening blood spots, but essentially normal urine excretion of EMA and isobutyrylglycine, accumulated increased levels of isobutyrylcarnitine in the cell medium compared with normal controls (Table 2). In two of these cell lines (Table 2, Patients 2 and 8), a mildly increased accumulation of butyrylcarnitine was noted. Urine was available from one of these patients and revealed a borderline normal excretion of EMA (19 $\mu \mathrm{g} / \mathrm{mg}$ creatinine; reference range: $<20$; Table 2, Patient 8 ). Based on our experience, such patients are either homozygous or compound heterozygous for one or two common SCAD gene variants, $625 \mathrm{G}>\mathrm{A}$ and $511 \mathrm{C}>\mathrm{T}$. Indeed, molecular genetic analysis of the SCAD gene in DNA extracted from cell cultures of both patients revealed that they are homozygous for the $625 \mathrm{G}>\mathrm{A}$ variant that has an allelic frequency of $22 \%$ in the general population. ${ }^{14}$ Fibroblast cultures of available SCADdeficient cases $(\mathrm{n}=14)$ accumulated abnormal concentrations of butyrylcarnitine (Table 3 ).

\section{Molecular genetic analysis}

A total of 10 different genetic alterations leading to missense mutations in ACAD8 were identified in nine of our cases (11 families, Table 1). Six infants were compound heterozygous for two mutations and three patients in three unrelated families were homozygous for three different mutations. Three infants of European descent were found to carry the same allele (455T $>$ C, M130T in the mature protein). Interestingly, all three patients with the $455 \mathrm{~T}>\mathrm{C}$ allele also had a putative splice-site mutation near exon $3,+3 \mathrm{~A}>\mathrm{G}$ (Table 1), an alteration of unknown significance that may only be linked to the protein-encoding lesion or may be the alteration leading to the observed biochemical phenotype. Another allele common to three unrelated patients was $1129 \mathrm{G}>\mathrm{A}, \mathrm{G} 355 \mathrm{~S}$ in the mature protein (Table 1), which alters a residue conserved among four ACD proteins including SCAD as well as medium-chain acylCoA dehydrogenase (MCAD), short/branch-chain acyl-CoA dehydrogenase (SBCAD), and isovaleryl-CoA dehydrogenase (IVD)..$^{20}$ One of the three patients carrying the $1129 \mathrm{G}>\mathrm{A} \mathrm{mu}-$ tation (Patient 11) was only heterozygous for this mutation without another alteration found in any other exon or intronic splice sites. The mild biochemical phenotype observed for Patient 11 from cell studies, and plasma and urine analysis suggests that this infant is at minimum a carrier for IBD deficiency. However, given the focused approach of our molecular genetic analysis, the presence of a promoter mutation, large deletion, or splice-site alteration undetectable by direct gene sequencing cannot be ruled out.

\section{Urine acylcarnitines}

Urine acylcarnitine analysis was performed on all available samples from IBD- $(\mathrm{n}=8)$ and SCAD- $(\mathrm{n}=12)$ deficient cases, as well as the IBD carrier (Patient 11, Table 2). The excretion of $\mathrm{C}_{4}$-acylcarnitine was elevated (range: 3.48-57.62 $\mathrm{mmol} / \mathrm{mol}$ creatinine; median: 20.30; reference range: $<3.00$; Table 2) in all IBD-deficient patients and in three patients $(\mathrm{Pa}-$ tients 8,12 , and 13, Table 3) with SCAD deficiency (range: $3.7-11.36 \mathrm{mmol} / \mathrm{mol}$ creatinine; median: $4.40 \mathrm{mmol} / \mathrm{mol} \mathrm{cre-}$ atinine; Table 3). Further discrimination between controls, IBD-deficient, and SCAD-deficient cases was achieved by comparing the ratio of $\mathrm{C}_{4}$-acylcarnitine to $\mathrm{C}_{3}$-acylcarnitine. Recursive partitioning was used to find the optimal values of $\mathrm{C}_{4}$-acylcarnitine and the $\mathrm{C}_{4} / \mathrm{C}_{3}$-acylcarnitine ratio for discriminating among normal, IBD-deficient, and SCAD-deficient patients with two mutated alleles. ${ }^{19}$ Exact binomial confidence limits are given for the sensitivity and specificity estimates. The cutoff for $\mathrm{C}_{4}$-acylcarnitine was set at $3.00 \mu \mathrm{mol} / \mathrm{L}(100 \%$ sensitivity [95\% confidence interval (CI): 63.1-100\%]; $83.6 \%$ specificity [95\% CI: 77.2-88.8\%]) and that for the $\mathrm{C}_{4} / \mathrm{C}_{3}$ acylcarnitine ratio was set at 24 (100\% sensitivity [95\% CI: 63.1$100 \%$ ]; $99.4 \%$ specificity [ $95 \%$ CI: $96.8-100.0 \%$ ]). The use of both cutoff values together allowed IBD-deficient cases with two mutated alleles to be differentiated from SCAD-deficient patients and controls with $100 \%$ sensitivity (95\% CI: 63.1$100 \%$ ) and $100 \%$ specificity (95\% CI: $97.9 \%-100 \%$ ) (Fig. 3). 








\section{Urine EMA}

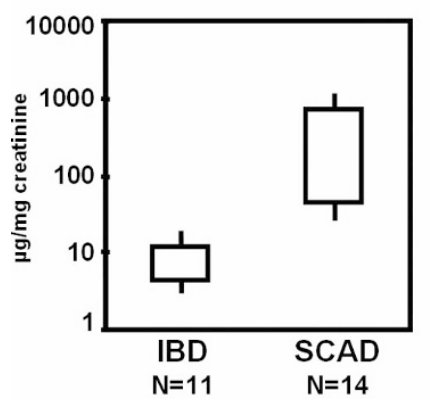

D. Urine IBG

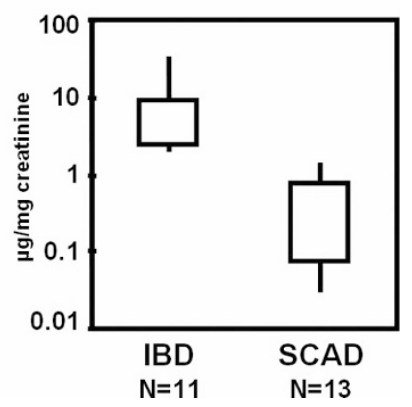

Fig. 2. Box plots of biomarker concentration distributions for isobutyryl-CoA dehydrogenase (IBD)- and short-chain acyl-CoA dehydrogenase (SCAD)-deficient patients. (A) $\mathrm{C}_{4}$-acylcarnitine concentration in newborn-screening blood spots $\left(\mathrm{DBS} \mathrm{C}_{4}\right)$. $(\mathrm{B}) \mathrm{C}_{4}$ acylcarnitine concentration in plasma. (C and D) Concentration of ethylmalonic acid (EMA) and isobutyrylglycine (IBG) in urine, respectively. For C and D the y-axis is plotted on a log scale. The vertical lines indicate maximum and minimum values; the upper and lower end of the boxes correspond to the 90th and 10th percentiles, respectively.

Thus, unlike the isobutyrylglycine excretion, the analysis of urine acylcarnitines appears to be a practical tool for diagnosing IBD deficiency.

\section{DISCUSSION}

Until this report, IBD deficiency was identified in only nine patients worldwide. However, based on our newborn screening experience, the incidence of this autosomal recessive condition is at least 1:70,000 livebirths. The index patient presented at 12 months with anemia, a dilated cardiomyopathy, and low plasma carnitine levels. With L-carnitine supplementation, this patient's symptoms resolved, and she remains asymptomatic at almost 11 years of age (Dr. Steven Cederbaum, 2006, Mental Retardation Research Center, UCLA, Los Angeles, CA, personal communication). The hallmark of this defect in valine metabolism is an elevated intramitochondrial accumulation of isobutyryl-CoA that is shuttled outside the cell after esterification to isobutyrylcarnitine. The latter is detectable in plasma, urine, and blood spot samples as a $\mathrm{C}_{4}$-acylcarnitine that also represents an isomer, butyrylcarnitine, when analyzed using standard MS/MS-based procedures for acylcarnitine analysis. ${ }^{17}$ It is not surprising, then, that the patients described here and eight of the nine previously reported patients were identified through newborn screening using MS/MS for acylcarnitine analysis. This also highlights the im-

portance of considering the differential diagnosis of isolated $\mathrm{C}_{4}$-acylcarnitine elevations when choosing appropriate follow-up investigations for the evaluation of newborns who present with such abnormalities by newborn screening. The majority of newborn screening laboratories request a repeat blood spot when encountering an abnormal result. However, in our experience, this follow-up approach will only enable the designation of a rare initial false-positive screening result at the expense of delaying the correct diagnosis of a child with a persistently elevated $\mathrm{C}_{4}$-acylcarnitine.

Although IBD deficiency can be diagnosed in cultured fibroblasts, there is considerable aversion to obtaining skin biopsies from apparently asymptomatic neonates. Moreover, the delay in obtaining results from fibroblasts due to culturing time can be lengthy. A less invasive and more rapid approach to diagnosing infants with positive newborn screening results is therefore preferable. Molecular genetic analysis of the ACAD8 gene is possible using blood samples, but is available only in research laboratories and with long turnaround times. A biochemical diagnostic marker for IBD deficiency is urinary isobutyrylglycine. However, as demonstrated in our patients and previously in others, this marker is unreliable., ${ }^{2,5}$ Therefore, we determined the diagnostic potential of urine acylcarnitine analysis in this context. Acylcarnitine analysis was originally developed for the diagnosis of organic acidurias in urine samples, but this specimen lost favor when it became clear that results in patients with fatty acid oxidation disorders were inconsistent. ${ }^{21-23}$ Plasma and dried blood spots have since become the preferred specimen for acylcarnitine analysis. However, in recent years, urine acylcarnitine analysis has reemerged as a useful screening tool in some situations. ${ }^{24}$ It appears to be of particular diagnostic value in the evaluation of patients with possible organic acidurias but inconclusive or minimally abnormal urine organic acid and plasma acylcarnitine profiles. In our IBD-deficient cohort, urine acylcarnitine analysis revealed an elevated $\mathrm{C}_{4}$-acylcarnitine excretion in all patients. Among 12 infants with SCAD deficiency, only three excreted elevated levels of $\mathrm{C}_{4}$-acylcarnitine (Table 3). Further discrimination between control, IBD deficient-, and SCAD-deficient infants was achieved by comparing the ratio of urine levels of $\mathrm{C}_{4}$-acylcarnitine to $\mathrm{C}_{3}$-acylcarnitine. The use of urine $\mathrm{C}_{4}$-acylcarnitine levels together with the urine $\mathrm{C}_{4} / \mathrm{C}_{3}$-acylcarnitine ratio allowed IBD-deficient cases with two mutated alleles to be differentiated from SCAD-deficient patients and controls with $100 \%$ sensitivity and $100 \%$ specificity (Fig. 3). Thus, unlike the determination of the isobutyrylglycine excretion, urine acylcarnitine analysis appears to be a practical tool for diagnosing IBD deficiency.

Molecular genetic analysis of ACAD 8 revealed a total of 10 different missense mutations in nine of our cases (Table 1). Four mutations, $443 \mathrm{C}>\mathrm{T}, 455 \mathrm{~T}>\mathrm{C}, 958 \mathrm{G}>\mathrm{A}$, and $988 \mathrm{C}>\mathrm{T}$, identified in our cohort have been described previously. ${ }^{5,20}$ Among the seven known mutations, five are alterations causing missense mutations and two result in the introduction of premature stop codons. $4,5,20$ We found five infants who were compound heterozygous for two mutations and three patients in three unrelated families who were homozygous for three different private mutations. Of note, one case (Patient 11, Ta- 
Table 3

Biochemical phenotypes of SCAD patients identified by newborn screening

\begin{tabular}{|c|c|c|c|c|c|c|c|}
\hline Patient & $\begin{array}{c}\text { NBS } \\
\mathrm{C}_{4} \text {-acylcarnitine } \\
(\mu \mathrm{mol} / \mathrm{L})\end{array}$ & $\begin{array}{c}\text { Plasma } \\
\mathrm{C}_{4} \text {-acylcarnitine } \\
(\mu \mathrm{mol} / \mathrm{L})\end{array}$ & $\begin{array}{c}\text { In vitro } \\
\text { probe } \mathrm{C}_{4} \text {-acylcarnitine } \\
(\mu \mathrm{mol} / \mathrm{g} \text { protein })\end{array}$ & $\begin{array}{c}\text { Urine EMA } \\
(\mu \mathrm{g} / \mathrm{mg} \text { creatinine })\end{array}$ & $\begin{array}{c}\text { Urine } \\
\text { isobutyrylglycine } \\
(\mu \mathrm{g} / \mathrm{mg} \text { creatinine })\end{array}$ & $\begin{array}{c}\text { Urine } \\
\mathrm{C}_{4} \text {-acylcarnitine } \\
(\mathrm{mmol} / \mathrm{mol} \text { creatinine })\end{array}$ & $\begin{array}{c}\text { Urine } \\
\mathrm{C}_{4} / \mathrm{C}_{3} \text { ratio }\end{array}$ \\
\hline 1 & 1.8 & 2.85 & 0.206 & 163.19 & 0.11 & ND & ND \\
\hline 2 & NA & ND & 0.210 & 46.74 & 0.31 & 0.96 & 2 \\
\hline 3 & NA & 0.56 & 0.240 & 25.15 & 0.06 & ND & ND \\
\hline 4 & NA & ND & 0.321 & 52.61 & ND & ND & ND \\
\hline 5 & NA & 2.71 & 0.345 & 1182.93 & 0.11 & 1.65 & 28 \\
\hline 6 & NA & ND & 0.353 & 215.63 & 0.39 & 1.31 & 7 \\
\hline 7 & NA & ND & 0.360 & ND & ND & ND & ND \\
\hline 8 & NA & 2.24 & 0.429 & 85.93 & 0.21 & 4.40 & 1 \\
\hline 9 & NA & 3.74 & 0.459 & ND & ND & ND & ND \\
\hline 10 & NA & ND & 0.566 & 213.96 & ND & 0.75 & 3 \\
\hline 11 & NA & 3.03 & 0.644 & 358.26 & 1.42 & 2.48 & 5 \\
\hline 12 & NA & 4.14 & 0.930 & 97.27 & 0.19 & 3.70 & 5 \\
\hline 13 & 1.6 & 2.34 & 0.959 & 85.94 & 0.21 & 11.36 & 2 \\
\hline 14 & NA & ND & 0.415 & 122.02 & 0.21 & 1.40 & 2 \\
\hline 15 & 2.9 & 4.8 & ND & 867.56 & 0.85 & 1.17 & 15 \\
\hline 16 & 1.6 & 0.53 & ND & 40 & 0.22 & 0.19 & 4 \\
\hline 17 & NA & 0.71 & ND & 77.98 & 0.03 & ND & ND \\
\hline 18 & NA & 2.26 & ND & 412.64 & ND & 2.47 & 9 \\
\hline Ref. range & $<1.5$ & $<1.06$ & $<0.125$ & $<20$ & $<11$ & $<3.00$ & $<24$ \\
\hline
\end{tabular}

EMA, ethylmalonic acid; NBS, newborn screening; NA, not available; ND, not determined for lack of specimen.

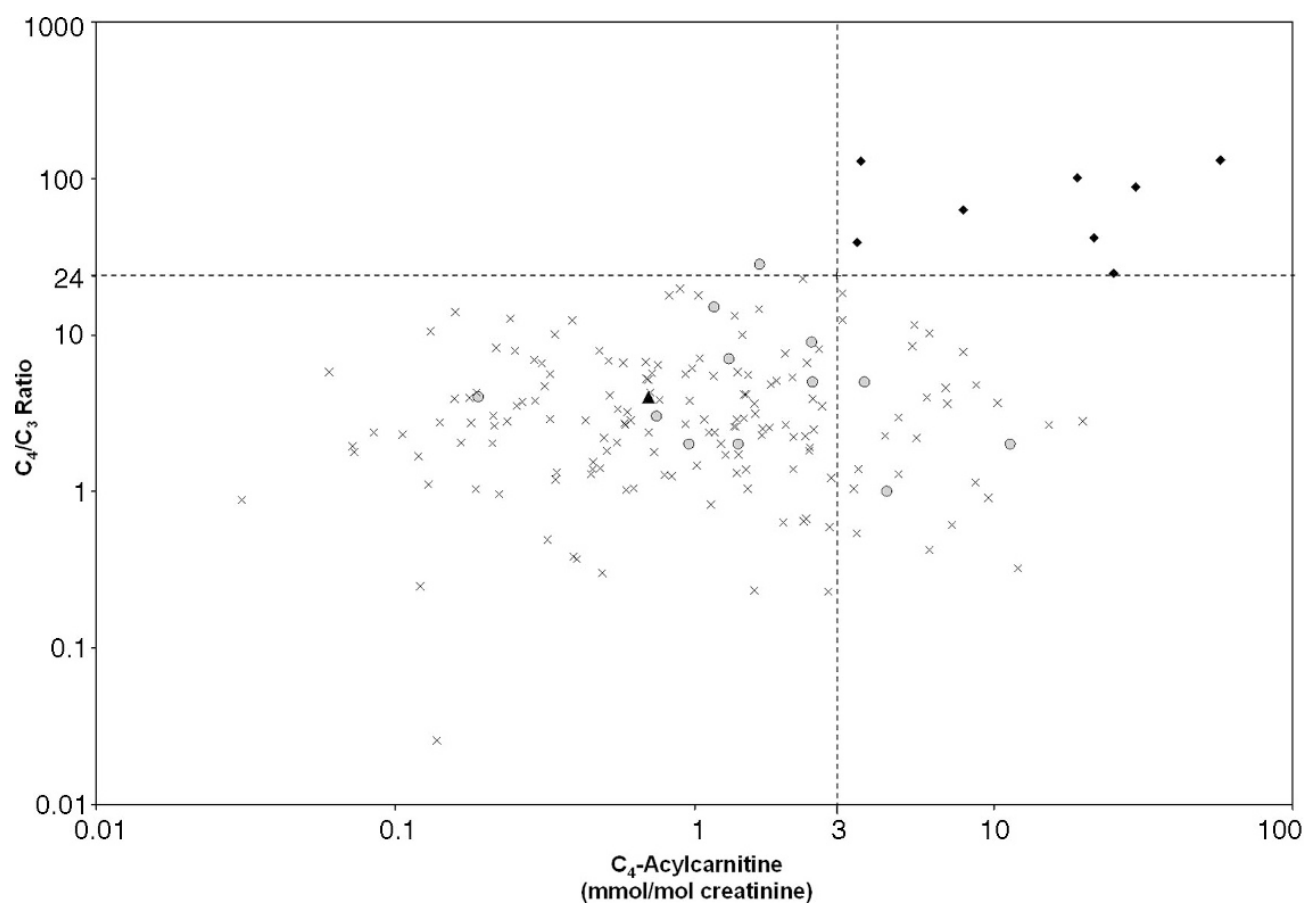

Fig. 3. Comparison of the urine $\mathrm{C}_{4}$-acylcarnitine concentration and the $\mathrm{C}_{4} / \mathrm{C}_{3}$ ratio in cases with isobutyryl-CoA dehydrogenase (IBD) $(n=8)$ and short-chain acyl-CoA dehydrogenase $(\mathrm{SCAD})$ deficiency $(\mathrm{n}=12)$. Filled diamonds represent values for IBD-deficient cases, shaded circles represent values for SCAD-deficient cases, crosses represent controls $(\mathrm{n}=160)$, and the filled triangle indicates values of the IBD carrier (Patient 11). Note that by applying cutoffs of $3 \mathrm{mmol} / \mathrm{mol}$ creatinine for $\mathrm{C}_{4}$-acylcarnitine and 24 for the $\mathrm{C}_{4} / \mathrm{C}_{3}$-acylcarnitine ratio (dotted lines), urine acylcarnitine analysis differentiates all cases with IBD deficiency (upper right quadrant) from SCAD-deficient cases and controls. Both axes are plotted on a log scale. 


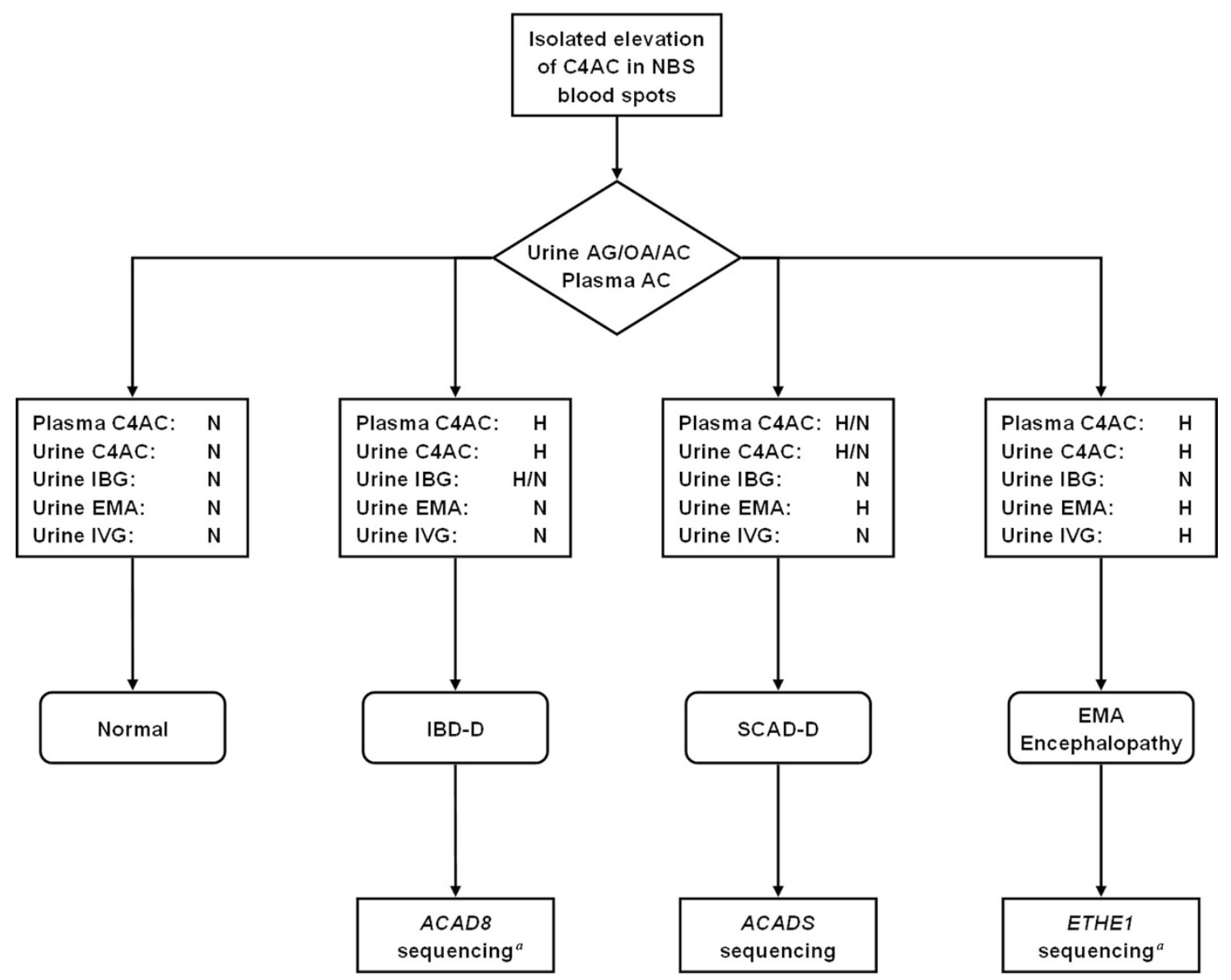

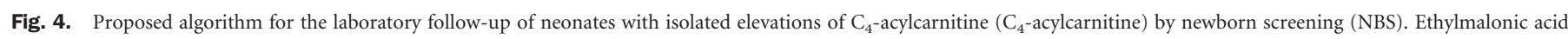

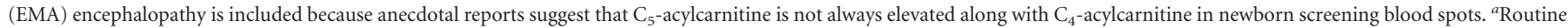


glycine; IVG, isovalerylglycine; N, normal; OA, organic acid analysis; SCAD-D, short-chain acyl-CoA dehydrogenase deficiency.

ble 1) was determined to have only one alteration, 1129G $>A$, after complete analysis of the exon and intragenic splice-site sequences of ACAD8. The biochemical phenotype of this patient is mild, follow-up plasma $\mathrm{C}_{4}$-acylcarnitine was abnormal and slightly above the median for IBD-deficient infants (1.87 $\mu \mathrm{mol} / \mathrm{L}$; median: $1.52 \mu \mathrm{mol} / \mathrm{L}$; Table 2 ), urine laboratory studies were unremarkable, and cell culture studies showed mild, although consistently abnormal, accumulations of isobutyrylcarnitine (Table 2). This patient presented with metopic craniosynostosis and a complete atrioventricular canal that prompted cytogenetic studies that revealed an abnormal karyotype [46,XY,add(18)(q23)]. Three infants of European ancestry shared one alteration, $455 \mathrm{~T}>\mathrm{C}$, suggesting that common IBD gene alleles may be prevalent among certain ethnic groups. However, the 455T $>C$ mutation was first observed in a patient described to be of Native American ancestry. ${ }^{5} \mathrm{We}$ found a potential splice-site mutation near exon $3(+3 \mathrm{~A}>\mathrm{G})$ in all our patients with the $455 \mathrm{~T}>\mathrm{C}$ alteration, a sequence variation not observed in the patient reported by Pedersen et al. ${ }^{5}$ Experiments to determine the significance of these sequence alterations on IBD expression, function, and stability are currently under way.
Based on our observations, we believe that the optimal, least invasive, and most rapid laboratory follow-up of an abnormal newborn screening result for an isolated elevation of $\mathrm{C}_{4}$-acylcarnitine includes plasma acylcarnitine, urine acylglycine, and urine acylcarnitine analyses following the algorithm depicted in Figure 4. This approach may also differentiate ethylmalonic encephalopathy, a lethal condition due to mutations in ETHE1, which in one case has been diagnosed after the finding of an isolated elevation of $\mathrm{C}_{4}$-acylcarnitine by newborn screening (U. Caruso, 2006, Ospedale Gaslini, Genoa, Italy, personal communication). ${ }^{25}$ Further confirmation of the diagnosis relying on molecular genetic or cell culture-based biochemical analysis will only be necessary in rare cases with equivocal results as observed in our two patients with IBD deficiency and homozygosity for SCAD gene variants.

Clinically, the patients reported here have done well without the prescription of a valine-restricted diet. L-Carnitine supplementation has been irregular, with normal carnitine levels documented in several patients without additional supplementation. These patients are now as old as 5 years of age. One patient was repeatedly hospitalized for IV hydration during her first 2 years of life due to vomiting and dehydration with otherwise mild intercurrent illnesses such as gastroenteritis and otitis 
media. During the first such episode, her plasma carnitine levels were found to be moderately reduced (total carnitine: 29 $\mu \mathrm{mol} / \mathrm{L}$, reference range: $35-84 \mu \mathrm{mol} / \mathrm{L}$; free carnitine: 13 $\mu \mathrm{mol} / \mathrm{L}$, reference range: $34-63 \mu \mathrm{mol} / \mathrm{L}$ ), and L-carnitine supplementation was initiated.

In conclusion, the natural history of IBD deficiency and SCAD deficiency is uncertain, leading the American College of Medical Genetics' Newborn Screening Expert Group to classify these conditions as secondary targets of the recommended universal newborn screening panel. ${ }^{15}$ Thus far, most IBD-deficient cases that have been identified by newborn screening have remained asymptomatic, suggesting that IBD deficiency is a relatively benign condition. This observation is likely to instigate consideration of its exclusion from newborn screening panels as was recently proposed for SCAD deficiency. ${ }^{9}$ However, based on the frequent episodes of dehydration in one of our patients and on the clinical presentation observed in the first IBD-deficient patient described, it seems possible that this disorder carries a risk for clinically relevant symptoms. Thus, it is prudent to remain vigilant for such episodes in infants identified with IBD deficiency through newborn screening and to continue to follow them with care. Only through long-term follow-up will it be possible to achieve evidencebased newborn screening for the benefit of all neonates, their families, and the societies into which they are born. We believe that application of the algorithm described here will aid in this process by allowing efficient and effective elucidation of isolated elevations of $\mathrm{C}_{4}$-acylcarnitine in newborn screening blood spots.

\section{ACKNOWLEDGMENTS}

J.V. was supported in part by NIH grant R01DK54936 and by the Pennsylvania State Tobacco Settlement Fund. The authors are grateful to Bambi Anderson, Maureen Black, and Joseph Huey for expert technical assistance, Sandra Bryant for performing the statistical analyses, and the Mayo Clinic Molecular Genetics Laboratory for ACADS mutation analysis. They also thank all the families who consented to enroll their children in this study and Drs. Piero Rinaldo and Harvey Levy for critical review of the manuscript.

\section{References}

1. Nguyen TV, Andresen Corydon TJ, Ghisla S, et al. Identification of isobutyryl-CoA dehydrogenase and its deficiency in humans. Mol Genet Metab 2002;77:68-79.

2. Roe CR, Cederbaum SD, Roe DS, Sweetman L, et al. Isolated isobutyryl-CoA dehydrogenase deficiency: an unrecognized defect in human valine metabolism. Mol Genet Metab 1998;65:264-271.

3. Koeberl DD, Young SP, Gregersen NS, Vockley J, et al. Rare disorders of metabolism with elevated butyryl- and isobutyryl-carnitine detected by tandem mass spectrometry newborn screening. Pediatr Res 2003;54:219-223.
4. Sass JO, Sander S, Zschocke J. Isobutyryl-CoA dehydrogenase deficiency: isobutyrylglycinuria and ACAD8 gene mutations in two infants. J Inherit Metab Dis 2004; 27:741-745.

5. Pedersen CB, Bischoff C, Christensen E, Simonsen H, et al. Variations in IBD (ACAD8) in Children with Elevated C4-Carnitine Detected by Tandem Mass Spectrometry Newborn Screening. Pediatr Res 2006;60:315-320.

6. Rinaldo P, Tortorelli S, Matern D. Recent developments and new applications of tandem mass spectrometry in newborn screening. Curr Opin Pediatr 2004;16:427433.

7. Amendt BA, Greene C, Sweetman L, Cloherty J, et al. Short-chain acyl-coenzyme A dehydrogenase deficiency. Clinical and biochemical studies in two patients. J Clin Invest 1987;79:1303-1309.

8. Bok LA, Vreken P, Wijburg FA, Wanders RJ, et al. Short-chain Acyl-CoA dehydrogenase deficiency: studies in a large family adding to the complexity of the disorder. Pediatrics 2003;112:1152-1155.

9. van Maldegem BT, Duran M, Wanders RJ, Niezen-Koning KE, et al. Clinical, biochemical, and genetic heterogeneity in short-chain acyl-coenzyme A dehydrogenase deficiency. JAMA 2006;296:943-952.

10. Corydon MJ Gregersen N, Lehnert W, Ribes A, et al. Ethylmalonic aciduria is associated with an amino acid variant of short chain acyl-coenzyme A dehydrogenase. Pediatr Res 1996;39:1059-1066.

11. Corydon TJ Bross P, Jensen TJ, Corydon MJ, et al. Rapid degradation of short-chain acyl-CoA dehydrogenase variants with temperature-sensitive folding defects occurs after import into mitochondria. J Biol Chem 1998;273:13065-13071.

12. Corydon MJ, Vockley J, Rinaldo P, Rhead WJ, et al. Role of common gene variations in the molecular pathogenesis of short-chain acyl-CoA dehydrogenase deficiency. Pediatr Res 2001;49:18-23.

13. Nguyen TV, Riggs C, Babovic-Vuksanovic D, Kim YS, et al. Purification and characterization of two polymorphic variants of short chain acyl-CoA dehydrogenase reveal reduction of catalytic activity and stability of the Gly185Ser enzyme. Biochemistry 2002;41:11126-11133.

14. Nagan N, et al. The frequency of short-chain acyl-CoA dehydrogenase gene variants in the US population and correlation with the $\mathrm{C}(4)$-acylcarnitine concentration in newborn blood spots. Mol Genet Metab 2003;78:239-246.

15. Watson MS, Lloyd-Puryear MA, Mann MY, Rinaldo P, Howell RR. Main report. Genet Med 2006;8:12S-252S.

16. Rinaldo $\mathrm{P}, \mathrm{Hahn} \mathrm{SH}, \mathrm{Matern} \mathrm{D}$. Inborn errors of amino acid, organic acid, and fatty acid metabolism. In: Ashwood ER, Bruns DE, Burtis CA, editors. Tietz textbook of clinical chemistry. Philadelphia: WB Saunders, 2005:2207-2247.

17. Matern D. Acylcarnitine analysis. In: Blau N, Duran M, Gibson KM, editors. Laboratory guide to the methods in biochemical genetics. Heidelberg: Springer-Verlag, 2006.

18. Lowry OH, Rosebrough NJ, Farr AL, Randall RJ. Protein measurement with the folin phenol reagent. J Biol Chem 1951;193:265-275.

19. Therneau TM, Atkinson EJ. An introduction to recursive partitioning using the RPART routines. Rochester, MN: Section of Biostatistics, Mayo Clinic, 1997.

20. Battaile KP, Nguyen TV, Vockley J, Kim JJ, et al. Structures of isobutyryl-CoA dehydrogenase and enzyme-product complex: comparison with isovaleryl- and short-chain acyl-CoA dehydrogenases. J Biol Chem 2004;279:16526-16534.

21. Chalmers RA, Roe CR, Stacey TE, Hoppel CL, et al. Urinary excretion of L-carnitine and acylcarnitines by patients with disorders of organic acid metabolism: evidence for secondary insufficiency of L-carnitine. Pediatr Res 1984;18:1325-1328.

22. Millington DS, Terada N, Chace DH, Chen YT, et al. The role of tandem mass spectrometry in the diagnosis of fatty acid oxidation disorders. Progr Clin Biol Res 1992;375:339-354.

23. Roe CR, Millington DS, Maltby DA, Bohan TP, Hoppel CL, et al. L-carnitine enhances excretion of propionyl coenzyme A as propionylcarnitine in propionic acidemia. J Clin Invest 1984;73:1785-1788.

24. Tortorelli S, Hahn SH, Cowan TM, Brewster TG, et al. The urinary excretion of glutarylcarnitine is an informative tool in the biochemical diagnosis of glutaric acidemia type I. Mol Genet Metab 2005;84:137-143.

25. Tiranti V, D'Adamo P, Briem E, Ferrari G, et al. Ethylmalonic encephalopathy is caused by mutations in ETHE1, a gene encoding a mitochondrial matrix protein. Am J Hum Genet 2004;74:239-252. 